\title{
Germanium-on-Silicon Planar Concave Grating Wavelength (de)multiplexers in the Mid-Infrared
}

\author{
Aditya Malik, ${ }^{1,2, a)}$ Muhammad Muneeb, ${ }^{1,2,3}$ Yosuke Shimura, ${ }^{4,5}$ Joris Van Campenhout, ${ }^{4}$ Roger Loo, ${ }^{4}$ and \\ Gunther Roelkens ${ }^{1,2,3}$ \\ 1) Photonics Research Group, Ghent University-imec, Sint-Pietersnieuwstraat 41, 9000 Ghent, \\ Belgium \\ 2) Center for Nano- and Biophotonics, Ghent University, Belgium \\ ${ }^{3)}$ COBRA Research Institute, Eindhoven University of Technology, P.O. Box 513, Eindhoven 5600 MB, \\ The Netherlands \\ 4) imec, Kapeldreef 75, 3001 Leuven, Belgium \\ ${ }^{5)}$ Instituut voor Kern- en Stralingsfysica, KU Leuven, 3001 Leuven, Belgium
}

(Dated: 12 August 2013)

Mid-infrared wavelength (de)multiplexers based on planar concave gratings (PCGs) fabricated on a germanium-on-silicon waveguide platform are presented. PCGs with two different types of gratings (flat facet and distributed bragg reflectors) are analyzed for both TE and TM polarization. The insertion loss and cross talk for flat facet PCGs are found to be -7.6/-6.4 dB and 27/21 dB for TE/TM polarization. For distributed bragg reflector PCGs the insertion loss and cross talk are found to be $-4.9 /-4.2 \mathrm{~dB}$ and $22 / 23 \mathrm{~dB}$ for TE/TM polarization.

PACS numbers: $42.79 . \mathrm{Sz}$

Keywords: mid-infrared; planar concave gratings

\section{INTRODUCTION}

The mid-infrared (Mid-IR) wavelength region $(3-12 \mu \mathrm{m})$ is of interest for spectroscopic sensing applications. This interest is related to the fact that many gases have a specific fingerprint absorption spectrum in this wavelength range, which opens up a wide range of applications ${ }^{1}$. In order to tap the full potential of this fingerprint region, rugged and handheld measurement systems are needed. In the past decade, there have been great advances in quantum cascade lasers (QCLs) and interband cascade lasers (ICLs) which have enabled on-chip coherent MidIR light generation. The development of a CMOS compatible Mid-IR waveguide platform, which would allow one to combine the interesting functionalities and cheap manufacturing provided by passive photonic integrated circuits with the QCL-based and ICL-based Mid-IR light sources would therefore be an appealing approach to realize midIR light engines. Typically distributed feedback laser sources are used which can be designed in an array, each emitting at a specific wavelength, which is tunable over a narrow wavelength range ${ }^{2}$. A very appealing component which can enable beam combining of these DFB arrays is a wavelength multiplexer. These multiplexers can also be used for separating a broadband Mid-IR light source in desired wavelength bands according to the requirements of the envisioned spectroscopic system. Recently, we have demonstrated wavelength (de)multiplexing in the Mid-IR (5.35 $\mu \mathrm{m}$ center wavelength) on the germanium-on-silicon platform using

a)Electronic mail: aditya.malik@intec.ugent.be arrayed waveguide gratings $(\mathrm{AWG})^{3}$. The insertion loss of the AWG was in the range of $-2 /-3 \mathrm{~dB}$ for TE/TM polarization and a cross talk of 20/16 dB was reported for a $5 \times 200 \mathrm{GHz}$ (18 nm channel spacing) AWG. For wavelength multiplexers, the figure of merit is the insertion loss of the device. However, for spectroscopic applications, it is desired that the cross talk of the device is reduced as much as possible. In this letter, we present a planar concave grating (PCG) (de)multiplexer with a much improved cross talk for both TE and TM polarization. It is known that for large channel spacing, PCGs are preferred over AWGs as the array waveguides in the AWG design start overlapping and sophisticated changes in design have to be made while for PCGs, the length of the free propagation region (FPR) decreases for large channel spacing making the device less prone to variations in free propagation region (FPR) layer thickness ${ }^{4}$. A PCG operating at $3.8 \mu \mathrm{m}$ has been demonstrated on the silicon-on-insulator (SOI) platform ${ }^{5}$ but as the underlying oxide starts absorbing heavily beyond $4 \mu \mathrm{m}$ wavelength $^{6}$, a PCG fabricated in germanium-on-silicon will allow spectroscopic systems to be designed over a large fraction of the mid-infrared wavelength range given that the transparency window of silicon ranges from 1.1 $\mu \mathrm{m}$ to $8 \mu \mathrm{m}$ and that of germanium ranges from $1.7 \mu \mathrm{m}$ to $14 \mu \mathrm{m}$. Germanium-on-silicon also seems interesting for butt coupling of QCL/ICL arrays with the photonic integrated circuits because of the relative low index contrast between germanium and silicon which improves the modal overlap and hence reduces the coupling losses between QCL/ICL and the passive waveguide. Combining this with waveguide losses in the range of $2.5-4 \mathrm{~dB} / \mathrm{cm}^{3}$, the germanium-on-silicon waveguide platform becomes a compelling platform for photonic integrated circuits in 
the mid-infrared.

\section{PCG DESIGN, FABRICATION AND CHARACTERIZATION}

A PCG combines the functionalities of two well-known optical components,s a diffraction grating to spatially separate (or combine) the wavelengths incident on it and a concave mirror to focus this light in one (or a series) of waveguides. Light is launched in a free propagating region (FPR) by an input waveguide. In the FPR, the light diffracts and hits the concave grating. The concave grating both diffracts (to spatially separate the light) and refocuses the light back in a series of output waveguides. Figure 1(a) shows a microscope picture of such a PCG, illustrating the different parts.

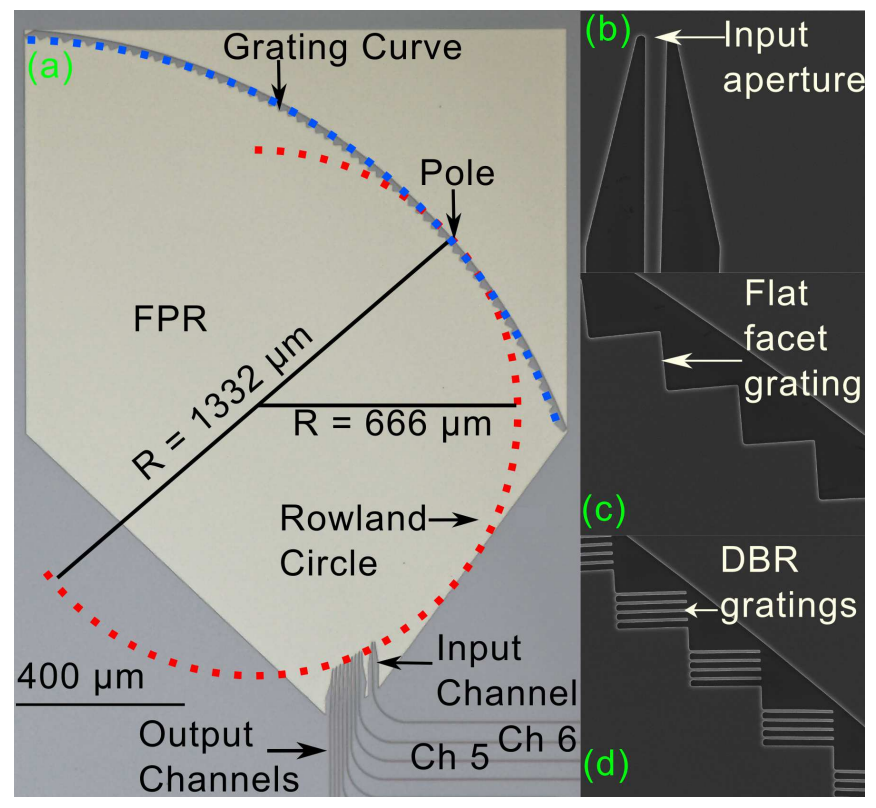

FIG. 1. (a) A microscopic image of the fabricated PCG illustrating the various parts, (b) an image of the input aperture, (c) an image of the fabricated flat facet grating and (d) an image of the fabricated DBR facet grating

The design presented in this paper is based on the Rowland geometry with one stigmatic point ${ }^{7}$. Each channel's response is simulated by scalar diffraction theory ${ }^{4}$. The single input and six output waveguides were placed on a circle of radius $666 \mu \mathrm{m}$ and the gratings were placed on a circle of radius $1332 \mu \mathrm{m}$. The input aperture was widened to a width of $4 \mu \mathrm{m}$ to launch light in the FPR and so were the output channel apertures. The channel spacing of the device was $25 \mathrm{~nm}$ and the footprint of the device was $1.5 \mathrm{~mm}$ by $1.2 \mathrm{~mm}$. The PCGs were fabricated in a $2 \mu \mathrm{m}$ thick germanium layer grown on 200 $\mathrm{mm} \mathrm{Si(001)} \mathrm{substrates.} \mathrm{A} \mathrm{metal} \mathrm{mask} \mathrm{was} \mathrm{defined} \mathrm{using}$ i-line contact lithography and liftoff. Germanium etching was then carried out in a $\mathrm{CF}_{4}: \mathrm{O}_{2}$ plasma in a $\mathrm{RIE}$ chamber. The germanium layer was completely etched through and the devices were air clad. For characterization of the devices, we coupled light from a commercially available QCL (Daylight solutions) in a single mode Indium Fluoride fiber (IRPhotonics). The cleaved end of the fiber was mounted on a piezo-controlled xyz stage to couple into the cleaved facet of the germanium-on-silicon waveguide. The output was collected from another piezomounted cleaved fiber and then sent to an InSb detector. To increase signal-to-noise ratio the output of the detector to a lock-in amplifier. The laser and lock-in amplifier were addressed together by software to simultaneously scan the wavelength and record the output ${ }^{3}$.

In this study, PCGs with two different grating facets were fabricated as described below.

\section{A. Flat facet grating}

In a first configuration flat germanium $(n=4) /$ air $(n=1)$ interface facets were used, leading to a Fresnel power reflection coefficient (assuming perpendicular incidence) of

$$
R=\left\{\frac{n_{1}-n_{2}}{n_{1}+n_{2}}\right\}^{2}=0.36
$$

Figure 1(c) shows an image of such fabricated flat facet grating. Figure 2(a) shows the normalized transmission of a six channel PCG for TE polarized light and Figure 2(b) shows that of TM polarized light. The normalization is carried out with respect to a straight waveguide, containing input and output taper and a straight waveguide of $0.5 \mathrm{~cm}$ length, present next to the device such that fiber-to-chip coupling losses are eliminated. The side lobe cross talk is around $27 / 21 \mathrm{~dB}$ for TE/TM polarization. To estimate the insertion loss of the device a gaussian fit of the response of the central channel for both polarizations was made. The insertion loss is $-7.6 /-6.4 \mathrm{~dB}$ for TE/TM polarization.

The insertion loss can be largely explained by the large Fresnel reflection loss of $-4.5 \mathrm{~dB}$ at the flat facet. The remaining $2-3 \mathrm{~dB}$ loss can be attributed to the nonverticality of the grating facets and lithography imperfections such as corner rounding. Figure 3 (a) and (b) show the normalized spectrum of the device for the TE and TM polarization respectively where each channel is displaced by an integer multiple of the channel spacing. It can be seen that the central lobe of the channels overlap nicely. Only the side lobes of the sixth channel are slightly worse compared to the others. This most likely comes from the fabrication imperfections of the grating facets such that they are reflecting back at wavelengths outside of the designated channel in the sixth output channel waveguide.

\section{B. DBR grating}

In the previous section we observed that although the cross talk of a six channel PCG is significantly better 


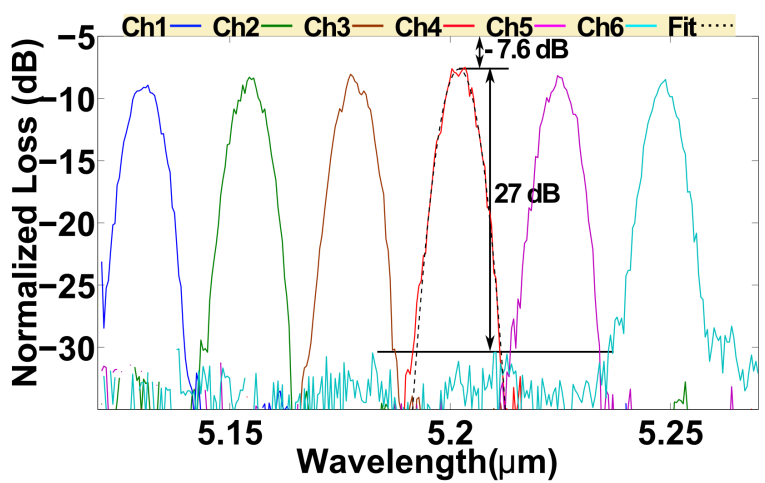

(a)

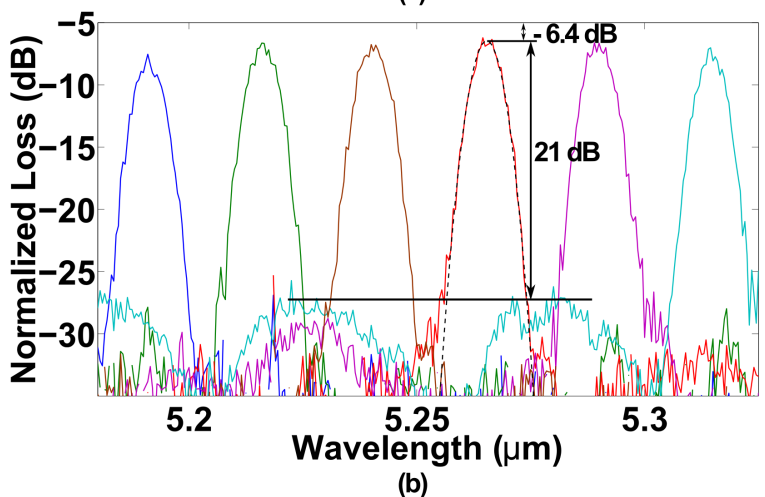

FIG. 2. Normalized spectra of a six channel PCG with flat grating facets for (a) TE polarized light and (b) TM polarized light.

than the reported $\mathrm{AWG}^{3}$, the insertion loss is still higher. One way of reducing insertion loss is to increase the reflection from the grating facets. DBR gratings can be used for that purpose. Normally one would like to fabricate DBRs which reflect all the light towards the output channel waveguides. However in fabrication we are limited to a minimum feature size of about $1 \mu \mathrm{m}$ due to i-line contact lithography. Therefore the period of DBR gratings was chosen to be $2.15 \mu \mathrm{m}$ and the fill factor $50 \%$. An image of the fabricated DBR grating can be seen in Figure 1(d). A 2-D simulation of the reflectivity of a four period DBR grating at a wavelength of $5.25 \mu \mathrm{m}$ was carried out as a function of grating period and fill factor as shown in Figure 4 (a) (for TE polarized light). The schematic at the top of the figure explains the dimensions of the gratings and the shaded area represents the combination of period and fill factor which will result in a minimum feature size larger than $1 \mu \mathrm{m}$. Figure 4 (b) shows the simulated reflection spectrum of the four period DBR grating with a period of $2.15 \mu \mathrm{m}$ and fill factor of 0.5 for both TE and TM polarized light in the $4-7 \mu \mathrm{m}$ wavelength range illustrating a peak reflectivity of $-1.45 /-1.25 \mathrm{~dB}$ and $-1 \mathrm{~dB}$ bandwidth of $2.1 / 1 \mu \mathrm{m}$ for $\mathrm{TE} / \mathrm{TM}$ polarization. The insertion loss is related to out of plane diffraction losses of the third order grating and can therefore not be improved by increasing the number of grating periods. Figure $5(\mathrm{a})$ shows the normalized
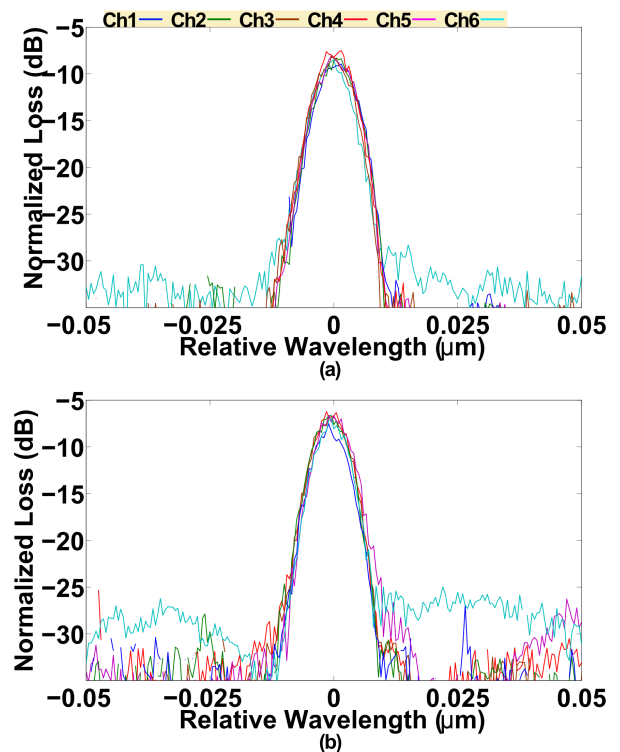

FIG. 3. Overlapped spectra of the six channel PCG with flat grating facets for (a) TE polarization and (b) TM polarization.

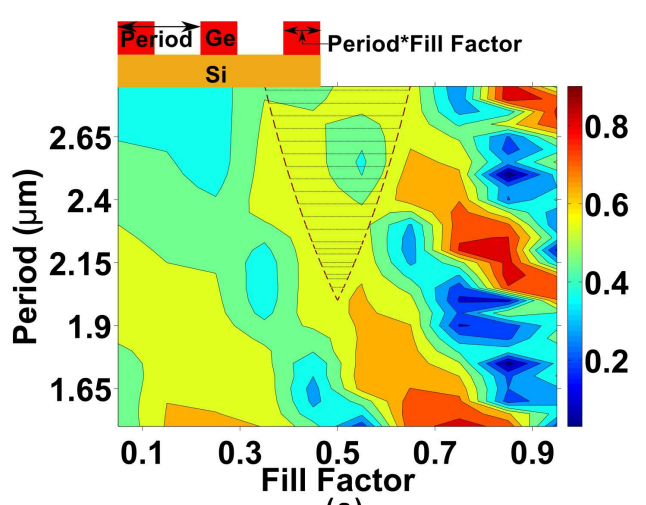

(a)

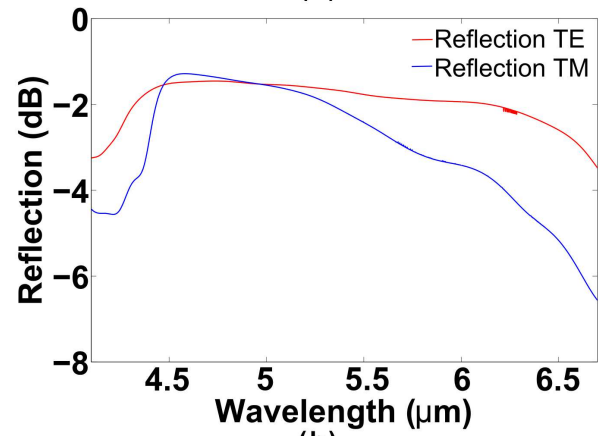

(b)

FIG. 4. (a) Simulated contour plot of reflectivity from a four period DBR grating as a function of period and fill factor. The shaded area shows the combination which can be fabricated using i-line contact lithography and the schematic at top explains grating parameters and (b) Simulated broadband reflection spectrum of a four period DBR grating with a period of $2.15 \mu \mathrm{m}$ and $50 \%$ fill factor for TE and TM polarized light. 


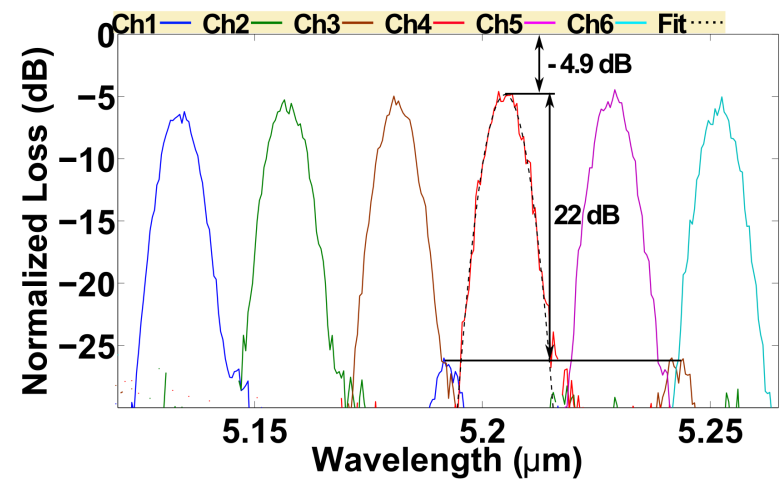

(a)

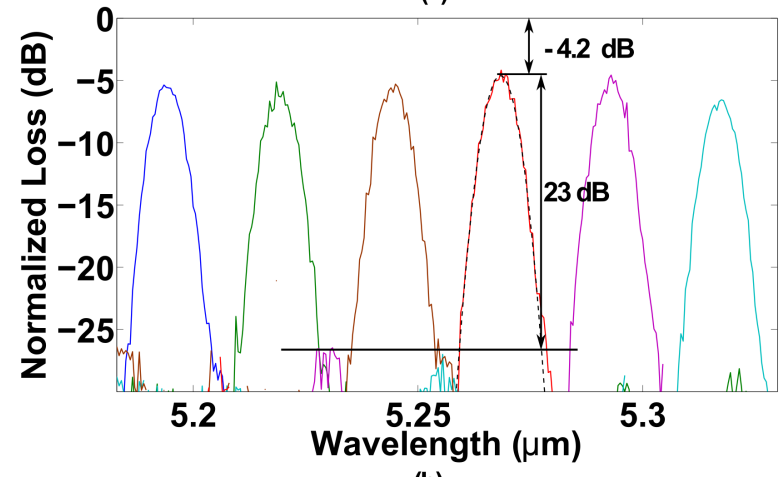

(b)

FIG. 5. Normalized spectra of a six channel PCG with DBR gratings for (a) TE polarized light and (b) TM polarized light.

transmission of a six channel PCG for TE polarized light and Figure 5(b) shows that of TM polarized light. The side lobe cross talk is around $22 / 23 \mathrm{~dB}$ for TE/TM polarization. To estimate the insertion loss of the device a gaussian fit of the response of the central channel for both polarizations was made. The insertion loss is $-4.9 /-$ $4.2 \mathrm{~dB}$ for TE/TM polarization. This clearly illustrates the insertion loss improvement of these devices by incorporating third order Bragg gratings. This result paves the way to achieving $<3 \mathrm{~dB}$ insertion loss when highly reflective gratings are used e.g. a period of $2.15 \mu \mathrm{m}$ and fill factor of $85 \%$ (320 nm spacing between grating teeth, which can be fabricated using standard CMOS tools) will result in $95 \%$ reflection as seen in Figure 4 (a).

Figure 6 (a) and (b) show the normalized spectrum of the device for TE and TM polarization respectively where each channel is displaced by an integer multiple of the channel spacing. Again only the sixth channel response suffers from slightly higher crosstalk similar to the case of the flat facet PCG.

\section{CONCLUSION}

We have demonstrated the use of germanium-on-silicon planar concave gratings as a wavelength (de)multiplexer for the mid-infrared. Although the insertion loss of the device is higher than our previously reported AWG based (de)multiplexer, the major cause for this is the lower reflection coming from the gratings, which can be substantially increased by using better performing gratings through the use of standard CMOS fabrication tools. The cross talk of these devices is comparable to PCGs fabricated on the SOI material platform at shorter wavelength in a CMOS pilot line. The integration of these devices with mid-infrared laser sources can lead to integrated mid-infrared laser light engines for spectroscopic sensing applications.

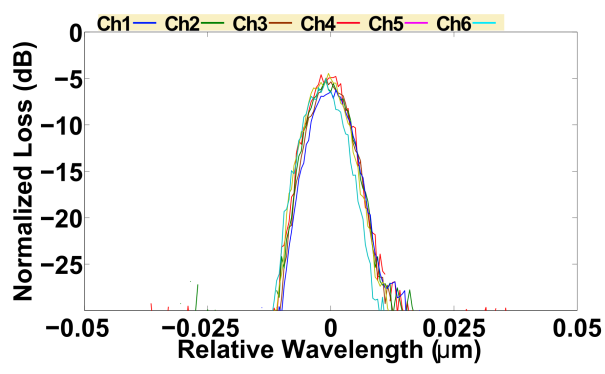

(a)

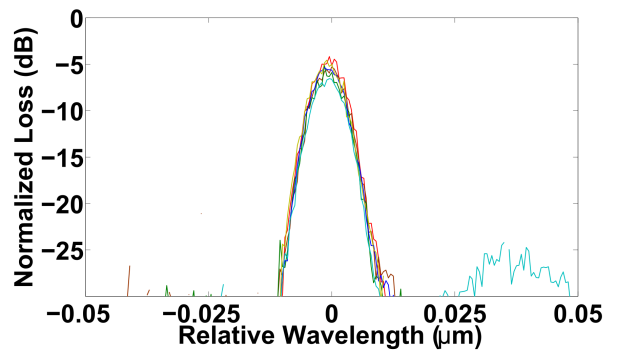

(b)

FIG. 6. Overlapped spectra of the six channel PCG with DBR gratings for (a) TE polarization and (b) TM polarization.

\section{ACKNOWLEDGMENTS}

This work was carried out in the framework of the FP-7 ERC project MIRACLE. Yosuke Shimura would like to acknowledge the FWO for a Pegasus Marie Curie scholarship.

${ }^{1}$ R.F.Curl et.al.,, "Quantum cascade lasers in chemical physics", Chem. Phys. Lett., Vol. 487, pp. 1 - 18 (2010).

${ }^{2}$ B.G.Lee et.al., "DFB Quantum Cascade Laser Arrays", IEEE J. Quant. Elec., Vol. 45, No. 5, pp. 554 - 565 (2009).

${ }^{3}$ A.Malik et.al., "Germanium-on-Silicon Mid-Infrared Arrayed Waveguide Grating Multiplexers", IEEE Phot.Tech.Lett, Volume:PP, Issue: 99, DOI:10.1109/LPT.2013.2276479.

${ }^{4} \mathrm{~K}$. A. McGreer, Diffraction from concave gratings in planar waveguides, IEEE Photonics Technology Letters, 7(3), 324-326 (1995).

${ }^{5}$ M.Muneeb et.al., "Demonstration of silicon on insulator midinfrared spectrometers operating at 3.8um", Opt. Exp., Vol.21 pp. 11659 - 11669 (2013)

${ }^{6}$ R.Soref, "Mid-infrared photonics in silicon and germanium", Nat. Phot., Vol. 4, pp. 495 - 497 (2010).

${ }^{7}$ R. Marz. Integrated Optics, Design and Modeling. (Artech House Inc., 1994). 